\title{
A classic reference updated
}

\author{
Reviewed by Jo Ellen Sherow, MPA, LATG
}

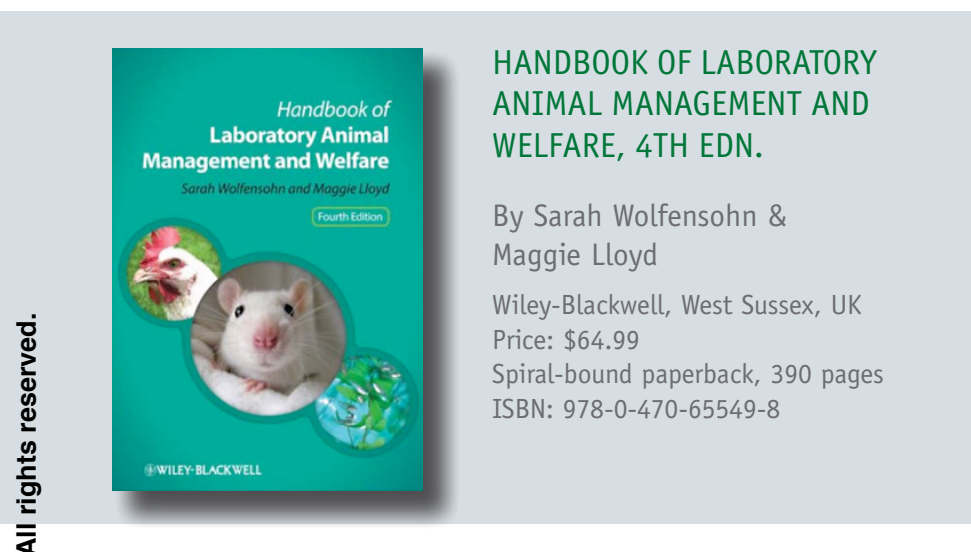

Now in its fourth edition, Handbook of Laboratory Animal Management and Welfare continues to be a valuable resource on a broad range of topics for laboratory animal care staff, IACUC members and research personnel. This latest edition offers updated content yet retains the depth of information for which the handbook is known. In addition, the book has been reorganized to improve its usefulness as a practical daily reference. The new edition also provides additional background information on environmental enrichment and updates to the sections on anesthesia and analgesia to reflect current best practices.

The introductory chapters cover basic animal welfare principles and ethical considerations for the use of animals in research. Although these chapters focus on European regulatory requirements for animal welfare, they contain enough generally applicable information to be of value to readers in other parts of the world as well. In addition, the increase in collaborative research spanning multiple jurisdictions necessitates a basic knowledge of other regulatory contexts, and this text provides an easily accessible overview of the European regulatory framework. Thoughtful sections address the importance of balancing animal welfare with science as well as ethical considerations for the use of animals in research.

The handbook is especially adept at weaving in applicable examples that can be adapted to any facility. Many examples of useful tools are provided, including scoring guides for general distress and for body condition in mice. Numerous illustrations throughout the book detail techniques used in various species. The photographs presented vary in quality and in some sections are only minimally useful; this volume therefore should not be seen as a substitute for more detailed instruction.

A chapter on animal handling and techniques presents a useful overview for those who are new to the subject and a refresher for those who are more experienced. With descriptions and illustrations of common techniques written in accessible language, as well as tables listing accepted administration volumes and needle gauge sizes for various species, it is an especially valuable reference for members of animal care and use review committees. The anesthesia and analgesia chapter similarly does an excellent job of providing information in a manner that can be easily digested by those new to the field. The surgery and surgical techniques section provides sufficient information to serve as an introduction to the subject; I would recommend this chapter to technicians preparing for certification who do not normally encounter surgery, because it covers a good amount of material in a very readable format.

The primary strength of this book continues to be its role as a comprehensive source of information on a wide range of animal species. Providing individual chapters on small laboratory animals, carnivores, primates, farm animals, wild animals and aquatic species, it is an ideal go-to reference for daily use. Each chapter contains sections on topics such as behavior, housing, handling and feeding as well as useful biological data reference material. The reader is also provided information on recognition of pain and distress in each species and any zoonotic issues that should be considered when working with the species. While no comprehensive book such as this one can provide all details needed to manage a species, it does a good job of providing the most pertinent information in an organized, readable format. As a reader working with a program that utilizes a wide range of species, I appreciated the inclusion of chapters on wild animals and aquatic species in the same volume as small laboratory species and farm animals. I also appreciated the inclusion of considerations for fish species other than zebrafish in the aquatic chapter. Although these chapters also focus on regulatory considerations outside of the US, they nevertheless provide excellent guidance on basic considerations such as how to calculate the dose or frequency of a test substance for a given species by extrapolating from a known dose rate or frequency.

This latest edition of Handbook of Laboratory Animal Management and Welfare improves upon previous versions and continues to be a worthwhile addition to the bookshelves of those working with laboratory animals. The primary audience for this book remains the entry-level technicians needing a comprehensive introduction to laboratory animal science. More experienced professionals will also appreciate it as an everyday reference that is well organized and easy to use. Though clearly not a substitute for clinical veterinary and research volumes or for more in depth training on techniques, the book provides a wealth of information and will no doubt continue to be a popular reference. 